\title{
Prevalência de astigimatismo refracional e sua relação com a acuidade visual de resolução em crianças de 2 a 36 meses
}

\author{
Refractionalastigmatism prevalence and its relationship with grating acuity \\ in children 2 to 36 months of age
}

\author{
César Lipener ${ }^{1}$ \\ Emilio de Haro Munoz ${ }^{2}$ \\ José Belmiro de Castro Moreira ${ }^{3}$ \\ Adriana Berezovsky ${ }^{4}$ \\ Solang'e Rios Salomão ${ }^{5}$ \\ Dora Fix Ventura ${ }^{6}$
}

\begin{tabular}{|l|}
\hline RESUMO \\
\hline Objetivo: A prevalência do astigmatismo refracional e sua relação com \\
a acuidade visual de resolução foram avaliados em amostra de crianças \\
pré-verbais. Métodos: Foram submetidas a exame oftalmológico 482 \\
crianças normais com idades variando entre 2 e 36 meses. Após exclusão \\
da amostra de 14 crianças com evidências de doenças oculares que \\
pudessem alterar a acuidade visual, permaneceram 468 crianças ( 936 \\
olhos), sendo 230 (49\%) do sexo masculino e 238 (51\%) do feminino. \\
Todas foram submetidas a exame de acuidade visual binoculare monocular \\
pelo procedimento do olhar preferencial através do método dos cartões \\
de acuidade de Teller. Foi realizado em todas as crianças exame de \\
refração sob cicloplegia e fundoscopia com oftalmoscópio indireto. \\
Resultados: O astigmatismo foi encontrado em 222 (47,43\%), crianças da \\
amostra, com predomínio do tipo hipermetrópico composto e da forma a \\
favor da regra em todas as faixas etárias. Em relação à magnitude, esta \\
ametropia foi maior ou igual a 1,00 dioptria cilíndrica em $24,35 \%$ dos \\
sujeitos, maior ou igual a 1,00 dioptria cilíndrica em $24,35 \%$ dos sujeitos, \\
maior ou igual a 2,00 dioptrias cilíndricas em $5,55 \%$ menor que 1,00 \\
dioptria cilíndrica em $26,92 \%$ e maior ou igual a 1,00 dioptria cilíndrica e \\
menor que 2,00 dioptrias cilíndricas em $18,73 \%$ A acuidade visual esteve \\
dentro da faixa de normalidade em 219 crianças da amostra, independen- \\
temente da magnitude, tipo e orientação do astigmatismo. Conclusão: A \\
acuidade visual de resolução medida pelo método dos cartões de acuidade \\
de Teller não foi influenciada pelo astigmatismo. \\
\hline
\end{tabular}

Descritores: Astigmatismo; Prevalência; Acuidade visual/métodos; Criança

\section{INTRODUÇ̃̃̃O}

O astigmatismo pode ser uma causa importante de deficiência visual na infância e sua detecção precoce tem grande valor clínico. À prescrição da correção óptica apropriada pode prevenir alterações no desenvolvimento da acuidade visual (AV) ${ }^{(1)}$. Vários autores relatam altas incidências de astigmatismo na infância e baixas na idade escolar, sugerindo que esta ametropia precoce praticamente desaparece entre o primeiro e o sexto ano de $\operatorname{vida}^{(2-8)}$.

A importância dos efeitos do astigmatismo no desenvolvimento da acuidade visual tem sido investigada e questionada ${ }^{(5)}$. A presença desta ametropia no primeiro ano de vida teria maior importância, pois este período é considerado crítico para o desenvolvimento do sistema visual humano. 
Nesta fase, haveria maior vulnerabilidade para ocorrência de deficiências visuais causadas por diferenças na qualidade de imagem nos diferentes meridianos, tendo como conseqüência uma variação meridional da visão observada em adultos ${ }^{(2,9-10)}$. A pouca cooperação e compreensão das crianças são consideradas como as principais dificuldades em se avaliar a acuidade pelos procedimentos clínicos habituais ${ }^{(11)}$. Mesmo com a simplificação dos métodos, a medida só é possível acima de dois anos e meio de idade. A técnica de determinação de acuidade visual pelo método do olhar preferencial baseia-se na observação de que bebês e crianças pré-verbais, quando defrontados com a escolha entre uma figura e um campo homogêneo, preferem olhar para a figura, mesmo que esta seja uma forma geométrica simples, como uma grade listrada, um estímulo comumente usado ${ }^{(12)}$.

A relação entre o erro refrativo e a AV medida pelo olhar preferencial em crianças de 2 a 12 meses tem sido estudada e, as mudanças observadas na acuidade não se mostraram correlatas às dos erros refrativos ${ }^{(13)}$. Uma forte relação entre o eixo do astigmatismo e o desenvolvimento de ambliopia com resultados mostrando que os astigmatismos oblíquos aumentam o risco de aparecimento desta condição tem sido relatada ${ }^{(14)}$.

Apesar do considerável número de estudos a respeito de astigmatismo refracional e acuidade visual, nenhum relaciona especificamente a ocorrência desta ametropia quanto ao tipo, eixo e magnitude e sua eventual relação com a visão em crianças pré-verbais.

O objetivo deste estudo é avaliar a prevalência de astigmatismo em crianças de 2 a 36 meses, quanto ao tipo, eixo e magnitude e analisar também sua relação com a acuidade visual medida pelo método dos cartões de acuidade de Teller.

\section{MÉTODOS}

\section{Sujeitos}

Foram examinadas 482 crianças, com idade entre 2 e 36 meses, sendo 237 do sexo masculino e 245 do sexo feminino provenientes de creches da rede municipal de São Paulo.

Os exames foram realizados nas próprias creches, por dois oftalmologistas e 6 ortoptistas dos Setores de Motilidade ocular extrínseca e Refração da Disciplina de Distúrbios Visuais Funcionais do Departamento de Oftalmologia da Escola Paulista de Medicina/UNIFESP. O consentimento informado verbal foi obtido dos pais e/ou responsáveis de cada criança envolvida no estudo. Este estudo seguiu os princípios da convenção de Helsinque.

Os critérios de inclusão foram: ausência de distúrbios neurológicos e nascimento a termo. Foi realizado exame oftalmológico completo em todas as crianças da amostra. Do total de 482 crianças examinadas, 14 foram excluídas da amostra, por apresentarem evidências de alterações oculares que pudessem comprometer a acuidade visual.

A amostra estudada foi então composta por 468 crianças
(936 olhos), 230 (49\%) do sexo masculino e 238 (51\%) do sexo feminino, com idades variando entre 2 e 36 meses.

Para a medida da acuidade visual foram utilizados os "cartões de acuidade de Teller" (Vistech Consultants Incorporation). Foi utilizado um conjunto de 16 pranchas em cor cinza (de 25,5x51,0 cm) com um orifício de observação central e uma grade de onda quadrada (de 12,5x12,5 cm) preta e branca ocupando a posição central, em apenas um dos lados. A refletância do cinza correspondia à média das refletâncias do preto e do branco da grade listrada. O teste é constituído por 16 cartões com grades de freqüência espacial diferentes, variando entre 0,23 e 38 ciclos/cm em intervalos aproximados de uma oitava $(0,32 ; 064 ; 1,3 ; 2,4 ; 4,8 ; 9,8 ; 19$ e 38 ciclos/cm).

A medida da acuidade visual seguiu o procedimento descrito no Teller Acuity Card Handbook ${ }^{(15)}$. O exame foi feito com a criança postada em frente ao painel cinza. Através da abertura existente neste, apresenta-se o primeiro cartão, iniciando-se com grades de baixa freqüência espacial de acordo com a idade e a distância do teste. $\mathrm{O}$ examinador avalia a resposta da criança ao teste e julga se a criança viu ou não a grade e se a confiabilidade das estimativas era boa.

Cada cartão é apresentado no mínimo duas vezes, uma vez com a grade à direita e outra à esquerda antes do julgamento. Em caso de dúvida, o cartão é reapresentado várias vezes quantas forem necessárias para a decisão. À medida que a criança vê uma grade, apresenta-se outra com freqüência espacial mais alta. A estimativa da acuidade visual é obtida por meio da grade mais fina que tenha sido julgada como reconhecida.

Para cada criança foram feitos três testes. O primeiro era binocular e os dois seguintes eram monoculares, com o uso de oclusores pequenos $\left(\mathrm{Oftam}^{\circledR}\right)$. A distância do teste era medida do orifício central do cartão aos olhos das crianças e mantida fixa. As crianças até 16 meses foram testadas a $38 \mathrm{~cm}$ e as outras a $55 \mathrm{~cm}$.

O exame oftalmológico foi realizado com retinoscópio em faixa portátil (Welch-Allyn), régua de esquiascopia (D. F. Vasconcelos), oftalmoscópio binocular indireto (Topcon modelo ID5) e lente asférica de +20D (Topcon). O exame compreendia: exame externo, avaliação da motilidade ocular extrínseca, refração e exame de fundo de olho. $\mathrm{O}$ exame refracional era realizado entre 30 a 40 minutos após a instilação de uma gota em cada olho de colírio cloridrato de ciclopentolato a $1 \%$. O exame fundoscópico foi feito através de oftalmoscopia binocular indireta.

Da amostra estudada (468 crianças), foram selecionadas para fins de análise aquelas que tinham astigmatismo. Foram consideradas astigmatas as crianças que apresentaram qualquer valor de astigmatismo em pelo menos um dos olhos. Estes foram divididos em quatro graus em função da sua magnitude em dioptrias cilíndricas (DC):

I. astigmatismo maior ou igual a 1,00 DC;

II. maior ou igual a 2,00 DC;

III. menor que $1,00 \mathrm{DC}$;

IV. maior que 1,00 e menor ou igual a $1,75 \mathrm{DC}$

Com a finalidade de comparação com a literatura, os astig- 
matas dos grupos I e II foram submetidos à análise estatística. É importante ressaltar que o grupo com astigmatismo maior ou igual a 1,00 DC incluiu também os olhos com valores maiores ou iguais a 2,00 DC.

Para avaliação do astigmatismo foi usada a convenção do cilindro positivo, tendo sido considerado apenas o valor do componente cilíndrico da ametropia. Os astigmatismos foram classificados quanto ao tipo em: astigmatismo hipermetrópico composto (AHC), astigmatismo hipermetrópico simples (AHS), astigmatismo miópico composto (AMC), astigmatismo miópico simples (AMS) e astigmatismo misto (AMI). Quanto à orientação foram considerados astigmatismos: a favor da regra (AFR), quando o eixo do cilindro está a $90^{\circ} \pm 15^{\circ}$; contra a regra (ACR) com eixo à $180^{\circ} \pm$ e oblíquo $(\mathrm{AO})$, com eixos entre $15^{\circ}$ e $75^{\circ} \mathrm{e}$ entre $105^{\circ}$ e $165^{\circ}$.

\section{RESULTADOS}

Na amostra estudada, foi encontrado astigmatismo em 222 crianças $(47,43 \%)$ e 398 olhos $(42,52 \%)$. As tabelas 1 e 2 mostram respectivamente o número de crianças e olhos por faixa etária e a prevalência de astigmatismo em cada grupo, considerando-se amostra total.

As figuras 1 e 2 mostram a prevalência do astigmatismo quanto ao tipo orientação em cada faixa etária, considerando apenas a amostra de crianças astigmatas $(\mathrm{N}=222)$.

\section{Crianças não astigmatas}

Do total de 468 crianças da amostra, 246 (52,56\%) não apresentaram astigmatismo, sendo que $202(43,16 \%)$ eram hipermétropes, $7(1,5 \%)$ eram míopes, 29 (6,2\%) não tinham

\begin{tabular}{|c|c|c|c|c|c|c|c|c|c|c|c|}
\hline \multirow{3}{*}{$\begin{array}{l}\text { Faixa etária } \\
\text { (intervalo } \\
\text { em meses) }\end{array}$} & \multirow{3}{*}{$\begin{array}{c}\text { Número } \\
\text { de crianças } \\
\text { por faixa }\end{array}$} & \multicolumn{10}{|c|}{ Crianças com astigmatismo } \\
\hline & & \multicolumn{2}{|c|}{ Total } & \multicolumn{2}{|c|}{$\geq 1,00$} & \multicolumn{2}{|c|}{$\geq 2,00$} & \multicolumn{2}{|c|}{$<1,00$} & \multicolumn{2}{|c|}{$1,00 \leq a<2,00$} \\
\hline & & $\mathbf{N}$ & $\mathrm{N} \%$ & $\mathbf{N}$ & $\mathrm{N} \%$ & $\mathbf{N}$ & $\mathrm{N} \%$ & $\mathbf{N}$ & $\mathbf{N} \%$ & $\mathbf{N}$ & $\mathbf{N} \%$ \\
\hline $1-3$ & 25 & 14 & 56,00 & 7 & 28,00 & - & - & 8 & 32,00 & 6 & 24,00 \\
\hline $4-6$ & 32 & 21 & 65,62 & 13 & 40,62 & 2 & 6,25 & 9 & 28,12 & 11 & 28,12 \\
\hline $7-9$ & 29 & 17 & 58,62 & 14 & 48,27 & 2 & 6,89 & 6 & 20,68 & 12 & 41,37 \\
\hline $10-12$ & 37 & 16 & 43,24 & 3 & 8,11 & 2 & 2,0 & 12 & 32,43 & 1 & 2,70 \\
\hline $13-15$ & 45 & 20 & 44,44 & 11 & 24,44 & 1 & 2,22 & 10 & 22,22 & 10 & 22,20 \\
\hline $16-18$ & 41 & 22 & 53,66 & 15 & 36,58 & 3 & 7,32 & 9 & 21,95 & 11 & 26,82 \\
\hline $19-21$ & 42 & 21 & 50,00 & 11 & 6,19 & 2 & 4,76 & 4 & 33,33 & 9 & 21,42 \\
\hline $22-24$ & 54 & 24 & 44,44 & 10 & 18,52 & 3 & 5,55 & 15 & 27,77 & 7 & 12,96 \\
\hline $25-27$ & 38 & 22 & 57,89 & 10 & 26,31 & 3 & 5,26 & 15 & 39,47 & 7 & 18,41 \\
\hline $28-30$ & 46 & 18 & 39,13 & 8 & 7,39 & 2 & 4,34 & 11 & 23,91 & 6 & 13,04 \\
\hline $31-33$ & 33 & 10 & 30,00 & 4 & 2,12 & 1 & 3,03 & 8 & 24,24 & 3 & 9,09 \\
\hline $34-36$ & 46 & 17 & 36,96 & 8 & 17,39 & 5 & 10,87 & 9 & 19,56 & 3 & 6,52 \\
\hline Total & 468 & 222 & 47,43 & 114 & 24,35 & 26 & 5,55 & 116 & 26,92 & 86 & 18,37 \\
\hline
\end{tabular}

\begin{tabular}{|c|c|c|c|c|c|c|c|c|c|c|c|}
\hline \multirow{3}{*}{$\begin{array}{l}\text { Faixa etária } \\
\text { (intervalo } \\
\text { em meses) }\end{array}$} & \multirow{3}{*}{$\begin{array}{l}\text { Total } \\
\text { de } \\
\text { olhos }\end{array}$} & \multicolumn{10}{|c|}{ Olhos astigmatas } \\
\hline & & \multicolumn{2}{|c|}{ Total } & \multicolumn{2}{|c|}{$\geq 1,00$} & \multicolumn{2}{|c|}{$\geq 2,00$} & \multicolumn{2}{|c|}{$<1,00$} & \multicolumn{2}{|c|}{$1,00 \leq a<2,00$} \\
\hline & & $\mathbf{N}$ & $\mathrm{N} \%$ & $\mathbf{N}$ & $\mathrm{N} \%$ & $\mathbf{N}$ & $\mathbf{N} \%$ & $\mathbf{N}$ & $\mathrm{N} \%$ & $\mathbf{N}$ & $\mathrm{N} \%$ \\
\hline $1-3$ & 50 & 25 & 50,00 & 11 & 22,00 & 0 & - & 14 & 28,00 & 11 & 22,00 \\
\hline $4-6$ & 64 & 39 & 60,93 & 25 & 39,06 & 4 & 6,25 & 14 & 21,87 & 21 & 32,81 \\
\hline $7-9$ & 58 & 34 & 58,62 & 25 & 43,10 & 4 & 6,89 & 9 & 15,51 & 21 & 36,20 \\
\hline $10-12$ & 74 & 25 & 33,78 & 6 & 8,10 & 4 & 5,40 & 19 & 25,67 & 2 & 2,70 \\
\hline $13-15$ & 90 & 36 & 40,00 & 21 & 23,33 & 2 & 2,22 & 15 & 16,66 & 19 & 21,11 \\
\hline $16-18$ & 82 & 42 & 51,21 & 27 & 32,92 & 5 & 1,09 & 15 & 18,29 & 20 & 24,39 \\
\hline $19-21$ & 84 & 39 & 46,42 & 17 & 20,23 & 3 & 3,57 & 22 & 26,19 & 14 & 16,66 \\
\hline $22-24$ & 108 & 43 & 39,81 & 18 & 16,66 & 5 & 4,62 & 25 & 23,14 & 13 & 12,03 \\
\hline $25-27$ & 76 & 38 & 50,00 & 15 & 19,73 & 4 & 5,26 & 23 & 30,26 & 11 & 14,47 \\
\hline $28-30$ & 92 & 32 & 34,78 & 5 & 16,30 & 4 & 4,34 & 17 & 18,47 & 11 & 11,95 \\
\hline $31-33$ & 66 & 17 & 25,75 & 6 & 9,09 & 1 & 1,51 & 11 & 16,66 & 5 & 7,57 \\
\hline $34-36$ & 92 & 28 & 30,43 & 14 & 15,21 & 9 & 9,78 & 14 & 15,21 & 5 & 5,43 \\
\hline Total & 936 & 398 & 42,52 & 192 & 21,36 & 45 & 4,80 & 198 & 21,15 & 153 & 16,34 \\
\hline
\end{tabular}


ametropia e $8(1,7 \%)$ tinham um olho emétrope e o outro míope ou hipermétrope.

\section{Acuidade visual (AV)}

A medida da $\mathrm{AV}$ foi realizada mono e binocularmente em todas as crianças, porém ao se fazer a análise dos resultados, considerou-se apenas os valores da acuidade monocular. Entre as 222 crianças astigmatas estudadas, três apresentaram

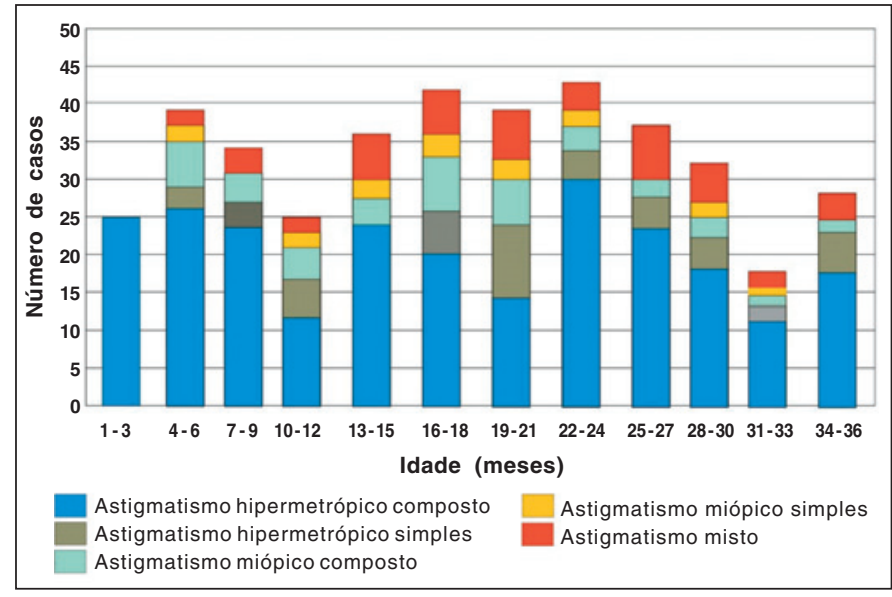

Figura 1 - Incidência dos 5 tipos de astigmatismo por faixa etária, considerando apenas amostra de astigmatas

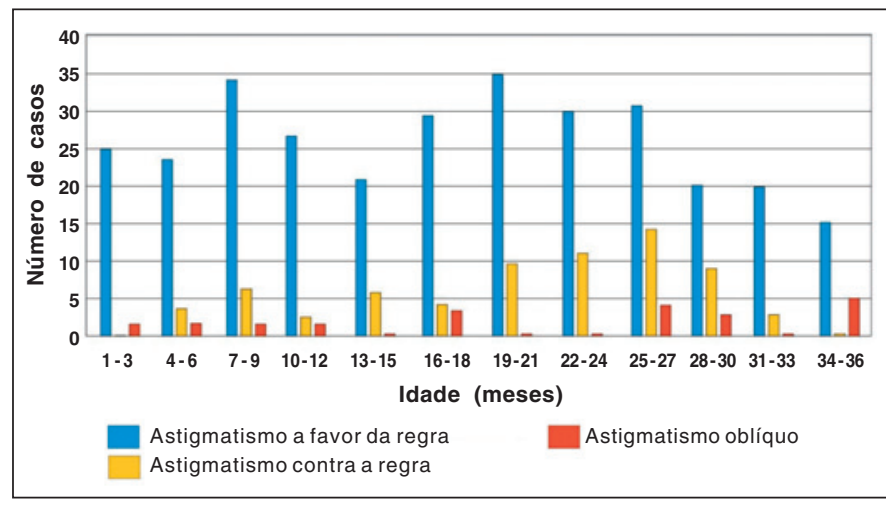

Figura 2 - Incidência de astigmatismo por orientação em cada faixa etária, considerando apenas amostra de astigmatas
AV abaixo da faixa de normalidade para a idade e uma era amblíope (apesar da AV dentro da faixa normal). A tabela 3 relaciona estes sujeitos.

\section{DISCUSSÃO}

Os resultados deste estudo revelaram alta prevalência de astigmatismo refracional em crianças até 36 meses de idade. Esta ametropia foi encontrada em 47,43\% da amostra, sendo que em $24,35 \%$ dos casos foi maior ou igual a 1,00 DC e em 5,55\%, maior ou igual a 2,00 DC, em concordância com os achados de outros autores ${ }^{(2-7)}$. A frequiência desta ametropia foi maior até os 9 meses com aparente diminuição a partir de 27 meses de idade (Tabela 1). Quando se leva em consideração apenas os valores maiores ou iguais a 1,00 DC, nota-se maior incidência até 9 meses e queda a partir de 25 a 27 meses, com exceção da faixa entre 10 e 12, que apresentou freqüência mais baixa.

Outros autores ${ }^{(16)}$ encontraram $29,7 \%$ de crianças com astigmatismo maior ou igual a 1,00 DC aos 12 meses de idade e $7,8 \%$ aos 42 meses de idade, o que foi interpretado por eles como uma diminuição progressiva na incidência desta ametropia com o crescimento. Outros estudos ${ }^{(5)}$ mostraram astigmatismo maior ou igual a $1,00 \mathrm{DC}$ em $36 \%$ das crianças entre 7 e 12 meses e $15 \%$ entre 12 e 15 meses de idade com $24,44 \%$ na faixa de 13 a 15 meses na presente amostra. Em relação aos casos em que a ametropia era maior ou igual a 2,00 DC, o mesmo estudo mostrou frequiência de 5,7\% em crianças até 15 meses, valor semelhante ao observado na presente amostra. Em um estudo ${ }^{(17)}$, ao fazer retinoscopia sob cicloplegia em crianças até 48 meses, os autores encontraram astigmatismo maior do que $1,00 \mathrm{DC}$ em $25 \%$ das crianças e acima de 2,00 DC em $3 \%$.

Em relação ao tipo de astigmatismo observado, houve predomínio do astigmatismo hipermetrópico composto em todas as faixas etárias, principalmente entre 1 e 3 meses, em que todas as crianças tinham esta forma de ametropia. $\mathrm{Na}$ literatura $^{(8)}$ observa-se a mesma situação em crianças de 1 e 4 anos de idade, embora haja referências sobre ${ }^{(3)}$ incidências maiores de astigmatismos miópicos simples e composto abaixo dos 5 meses de vida, com tendência para o tipo misto entre 5 e 8 meses e predomínio de hipermetrópico simples e composto após esta idade.

\begin{tabular}{|c|c|c|c|c|c|c|}
\hline Paciente & Sexo & $\begin{array}{l}\text { Idade } \\
\text { (meses) }\end{array}$ & $\begin{array}{l}\text { Valor do erro } \\
\text { refrativo }\end{array}$ & $\begin{array}{l}\text { Tipo de erro } \\
\text { refrativo }\end{array}$ & $\begin{array}{c}\text { AV } \\
\text { ciclos/grau }\end{array}$ & $\begin{array}{c}\text { Faixa normal de } \\
\text { AV para idade }\end{array}$ \\
\hline \multirow[t]{2}{*}{1} & M & 35 & OD 2,00 DC & $\mathrm{AHCO}$ & 9,6 & 13 a $38 \mathrm{c} / \mathrm{g}$ \\
\hline & & & OE 2,00 DC & $\mathrm{AHCO}$ & 9,6 & \\
\hline \multirow[t]{2}{*}{2} & M & 35 & OD $1,00 \mathrm{DC}$ & AHS FR & 4,7 & 13 a $38 \mathrm{c} / \mathrm{g}$ \\
\hline & & & OE $1,50 \mathrm{DC}$ & AHS O & 4,7 & \\
\hline \multirow[t]{2}{*}{3} & M & 30 & OD $3,50 \mathrm{DC}$ & AMI O & 4,7 & 9,6 a $26 \mathrm{c} / \mathrm{g}$ \\
\hline & & & OE $4,00 \mathrm{DC}$ & AMI FR & 3,1 & \\
\hline 4 & $F$ & 31 & OD $3,00 \mathrm{DC}$ & AMI FR & 13,0 & 9,6 a $26 \mathrm{c} / \mathrm{g}$ \\
\hline
\end{tabular}


A maior disparidade de resultados em relação a outros autores ocorreu quanto ao eixo do astigmatismo. Este estudo revelou que o tipo a favor da regra foi mais freqüente em todas as idades, com discreto aumento de incidências dos contra a regra entre 19 e 30 meses, porém sem evidências de inversão para esta orientação. Estes achados se chocam com a maioria dos trabalhos anteriores. Em outro estudo, o autor ${ }^{(18)}$ encontrou freqüência do tipo contra a regra duas vezes e meia maior do que a favor da regra em crianças cicloplegiadas abaixo de 3,5 anos, quando passou a predominar o astigmatismo a favor da regra; outros ${ }^{(17)}$ encontraram astigmatismo contra a regra em $56 \%$ das crianças até 48 meses, a favor da regra em $29 \%$ e oblíquo em $14 \%$.

Algumas diferenças entre os diversos estudos sobre esta ametropia, não só em relação à freqüência mas também à magnitude, tipo e eixo podem ser explicadas pelas diferentes metodologias e técnicas utilizadas (como por exemplo o uso ou não de cicloplegia) e pela sua própria fisiopatologia. Quanto ao uso ou não de drogas cicloplégicas, as opiniões divergem. Alguns trabalhos ${ }^{(19)}$ relatam a utilização da cicloplegia em sua amostra enquanto outros ${ }^{(20)}$ consideram o ciclopentolato preferível para cicloplegia em crianças por ser menos tóxico, ter efeito mais rápido e menor duração do efeito. No presente estudo, optou-se pelo uso de colírio de ciclopentolato a $1 \%$ por seu reconhecido poder cicloplégico, pela rapidez com que atinge o seu efeito máximo assim como droga de uso clínico freqüente e que pode facilitar a esquiascopia que é por vezes difícil em crianças.

Em outros estudos ${ }^{(18)}$ considerou-se a pressão das pálpebras sobre a córnea e a pressão exercida pelos músculos retos como fatores que explicariam a maior incidência de ACR e sua mudança para AFR em crianças devido à menor pressão das pálpebras sobre a córnea, à semelhança dos idosos e ao contrário de escolares e adultos, que teriam AFR com mais freqüência. O segundo fator seria a influência dos músculos retos, na forma o olho, predispondo a ACR pela pequena pressão palpebral. Estas hipóteses se confrontam com nossos resultados, já que neste estudo observou-se uma freqüência bem maior de AFR.

Outros fatores podem determinar uma variabilidade intrínseca dos erros astigmáticos: acomodação assimétrica nos diferentes meridianos, inclinação da lente, mudança da curvatura da córnea, a cicloforia, rotação compensatória do olho quando a cabeça é rodada e a extorsão binocular quando o foco muda de longe para perto ${ }^{(21-23)}$.

Há relatos que ${ }^{(24)}$ consideram que o astigmatismo total tem um componente externo, que seria a curvatura anterior da córnea, e quatro internos: curvatura posterior da córnea, superfícies anterior e posterior da lente, descentração do sistema óptico e astigmatismo refrativo da lente devido à variação da densidade óptica de suas várias camadas. Estas variáveis poderiam explicar alguns casos em que a AV é normal mesmo em crianças com valores significativos de astigmatismo refracional.

No presente estudo só foi considerado o astigmatismo refracional, que é na verdade a resultante óptica dos diversos componentes desta ametropia. Em função disto, é difícil fazer qualquer consideração a respeito da influência do astigmatismo corneano obtido pela ceratometria sobre a AV.

A acuidade visual esteve dentro da faixa normal para a idade na quase totalidade dos olhos astigmatas, independentemente da faixa etária e do valor do astigmatismo. Estes resultados estão de acordo com outros encontrados na literatura ${ }^{(25)}$, que não encontraram diferença significante na acuidade visual medida pelo método do olhar preferencial entre astigmatas e não astigmatas.

Em outras referências, os autores ${ }^{(26)}$ também concluíram da mesma forma e justificaram os resultados pela acomodação, que ocorreria de maneira diferente para listras horizontais e verticais de modo que nenhuma delas ficaria desfocada a ponto de diminuir a acuidade visual. Ao fazer a sobreposição dos valores individuais (olhos) de AV sobre a curva que relaciona idade e AV extraída do Teller Handbook ${ }^{(15)}$ nota-se que a maior parte dos olhos não só se encontra na faixa normal, mas principalmente acima dela. Tal fato, pode até sugerir que a curva de normalidade em nosso meio não corresponda àquela que foi proposta pelos primeiros trabalhos, e seja na verdade deslocada para cima, o que foi confirmado por outros autores $^{(12)}$ em estudo nacional.

Existe muita dúvida a respeito da época do desenvolvimento visual em que a presença do astigmatismo comprometeria a visão, ou permitiria a instalação da ambliopia meridional. Em um estudo, o autor ${ }^{(27)}$ mediu a melhor acuidade visual corrigida através de grades horizontais e verticais em crianças com idade entre 3 e 5 anos com altos astigmatismos e em crianças não astigmatas, tendo observado ambliopia significante entre as crianças astigmatas. Em outro relato na literatura ${ }^{(28)}$, examinouse um grupo de crianças entre 3 e 5 anos com uma alta prevalência de astigmatismo e encontrou ambliopia meridional entre as crianças com astigmatismo misto e com miópico simples e composto devido à acuidade reduzida para grades horizontais. As crianças com astigmatismo hipermetrópico simples e composto não apresentaram ambliopia meridional mas tiveram acuidade reduzida nas duas orientações de grades. A melhor acuidade de reconhecimento corrigida estava reduzida nos dois grupos sugerindo que a correção do astigmatismo deva ser feita antes dos 3 aos 5 anos para prevenir a ambliopia.

Os resultados nesta amostra sugerem que a AV medida através do método dos Cartões de Acuidade de Teller não é afetada pelo astigmatismo no primeiro ano de vida. Na amostra estudada a AV foi normal na grande maioria dos olhos independente do tipo de astigmatismo, de seu eixo ou de sua magnitude.

\section{CONCLUSÕES}

O presente estudo permitiu as seguintes conclusões:

1) A prevalência de astigmatismo em crianças de 3-36 meses de vida foi de 47,4. 
2) Em relação à magnitude, o astigmatismo foi maior ou igual a $1,00 \mathrm{DC}$ em $24,35 \%$, maior ou igual a 2,00 DC em 5,55\%, menor que $1,00 \mathrm{DC}$ em $26,92 \%$ e maior ou igual a $1,00 \mathrm{DC}$ e menor que $2,00 \mathrm{DC}$ em $18,37 \%$ das crianças examinadas.

3) Houve predomínio de astigmatismo do tipo hipermetrópico composto.

4) Em relação ao eixo, encontrou-se a forma a favor da regra com frequiência bem maior do que contra a regra ou oblíquo.

5) A acuidade visual medida pelo método dos cartões de acuidade de Teller não foi influenciada pelo astigmatismo.

\section{ABSTRACT}

Purpose: To evaluate refractional astigmatism prevalence and its relationship with grating acuity in a cohort of non-verbal children. Methods: 482 normal children, aged from 2 to 36 months, were submitted to ophthalmological examination. Fourteen subjects were excluded due to ocular disease and the sample remained with 468 subjects (936 eyes); 230 (49\%) males e 238 (51\%) females. Grating acuity was assessed binocularly and monocularly with Teller acuity cards. All children underwent eye examination including cycloplegic retinoscopy and fundus by indirect ophthalmoscopy. Results: Astigmatism was found in 222 (47.43\%) of the children, with the hyperopic and with the rule types most frequently found in all ages. Concerning magnitude, this condition was equal or greater than 1.00 cylindric diopter in $24.35 \%$ of the children; equal or greater than 2.00 cylindric diopter in $5.55 \%$; lower than 1.00 in $26.92 \%$ and between 1.00 and 2.00 in $18.73 \%$. Grating acuity was normal in 219 of the subjects, despite magnitude, type and orientation of astigmatism. Conclusion: Visual acuity assessed by the acuity card procedure was not influenced by astigmatism.

Keywords: Astigmatism; Prevalence; Visual acuity/methods; Child

\section{REFERÊNCIAS}

1. Banks MS, Salapatek P. Acuity and contrast sensitivity in 1-, 2-, and 3-monthold human infants. Invest Ophthalmol Vis Sci. 1978;17(4):361-5.

2. Howland HC, Atkinson J, Braddick O, French J. Infant astigmatism measured by photorefraction. Science. 1978;202(4365):331-3.
3. Mohindra I, Held R, Gwiazda J, Brill J. Astigmatism in infants. Science. 1978; 202(4365):329-31.

4. Banks MS. Infant refraction and accommodation. Int Ophthalmol Clin. 1980;20 (1):205-32.

5. Fulton AB, Dobson V, Salem D, Marc C, Petersen RA, Hansen RM. Cycloplegic refractions in infants and young children. Am J Ophthalmol. 1980;90(2): 239-47.

6. Gwiazda J, Scheiman M, Mohindra I, Held R. Astigmatism in children: changes in axis and amount from birth to six years. Invest Ophthalmol Vis Sci. 1984;25(1):88-92.

7. Tongue AC. Refractive errors in children. Pediatr Clin North Am. 1987;34(6): 1425-37. Review

8. Abrahamsson M, Fabian G, Sjostrand J. Changes in astigmatism between the ages of 1 and 4 years: a longitudinal study. Br J Ophthalmol. 1988;72(2):145-9.

9. Howland HC, Howland B. Photorefraction: a technique for study of refractive state at a distance. J Opt Soc Am. 1974;64(2):240-9.

10. Howland HC, Braddick O, Atkinson J, Howland B. Optics of photorefraction: orthogonal and isotropic methods. J Opt Soc Am. 1983;73(12):1701-8.

11. Dobson V, McDonald MA, Kohl P, Stern N, Samek M, Preston K. Visual acuity screening of infants and young children with the acuity card procedure. J Am Optom Assoc. 1986;57(4):284-9.

12. Salomão SR, Ventura DF. Does refractive error Influence visual acuity in early development? Am Orthopt J. 1995;45:97-107.

13. Kohl P, Rolen RD $3^{\text {rd }}$, Bedford AK, Samek M, Stern N. Refractive error and preferential looking visual acuity in human infants: a pilot study. J Am Optom Assoc. 1986;57(4):290-6.

14. Abrahamsson M, Sjostrand J. Astigmatic axis and amblyopia in childhood Acta Ophthalmol Scand. 2003;81(1):33-7.

15. Teller DY. Teller acuity card handbook. Dayton, OH: Vistech Consultants; 1989

16. Ingram RM, Barr A. Refraction of 1-year-old children after cycloplegia with $1 \%$ cyclopentolate: comparison with findings after atropinisation. Br J Ophthalmol. 1979;63(5):348-52.

17. Mayer DL, Hansen RM, Moore BD, Kim S, Fulton AB. Cycloplegic refractions in healthy children aged 1 through 48 months. Arch Ophthalmol. 2001; 119(11):1625-8

18. Dobson V, Fulton AB, Sebris SL. Cycloplegic refractions of infants and young children: the axis of astigmatism. Invest Ophthalmol Vis Sci. 1984;25(1):83-7.

19. Ingram RM, Walker C, Wilson JM, Arnold PE, Dally S. Prediction of amblyopia and squint by means of refraction at age 1 year. Br J Ophthalmol. 1986;70(1): $12-5$.

20. Moore AT. Refraction in childhood. Trans Ophthalmol Soc U K. 1985;104(Pt 6): 648-52.

21. Pascal JI. Intrinsic variability of astigmatic errors. Arch Ophthalmol. 1944;32: 123-4.

22. Hallden U, Henricsson M. Astigmatism of the lens by asymmetric contraction of the ciliary muscle. Acta Ophthalmol (Copenh). 1974;52(2):242-5.

23. Garzia RP, Nicholson SB. Clinical aspects of accommodative influences on astigmatism. J Am Optom Assoc. 1988;59(12):942-5. Review.

24. Anstice J. Astigmatism - its components and their changes with age. Am J Optom Arch Am Acad Optom. 1971;48(12):1001-6.

25. Gwiazda J, Mohindra I, Brill S, Held R. The development of visual acuity in infant astigmats. Invest Ophthalmol Vis Sci. 1985;26(12):1717-23.

26. Held R, Mohindra E, Gwiazda J, Bull S. Visual acuity of astigmatic infants and its meridional variation [abstract]. Invest Ophthalmol Vis Sci. 1977;17:65.

27. Harvey EM, Dobson V, Miller JM, Sherrill DL. Treatment of astigmatism-related amblyopia in 3- to 5- year-old children. Vision Res. 2004;44(14):1623-34.

28. Dobson V, Miller JM, Harvey EM, Mohan KM. Amblyopia in astigmatic preschool children. Vision Res. 2003;43(9):1081-90. 